\title{
Riesgo cardiovascular en población infantil con sobrepeso y obesidad
} Cardiovascular risk in infants overweight and obese

\author{
Jaime Pajuelo R. ${ }^{1,2}$, Hugo Arbañil H. ${ }^{1}$, José Sánchez G. ${ }^{1}$, Dante Gamarra G. ${ }^{1}$, \\ Lizardo Torres A. ${ }^{1}$, Rosa Pando A. ${ }^{1,2}$, Rosa Agüero Z \\ Servicio de Endocrinología del Hospital Nacional Dos de Mayo. \\ ${ }^{2}$ Instituto de Investigaciones Clínicas de la Facultad de Medicina UNMSM.
}

\begin{abstract}
Resumen
Introducción: El sobrepeso y la obesidad conducen a la aparición de comorbilidades como la diabetes mellitus tipo 2, hipertensión arterial, dislipidemias. Objetivos: Determinar la presencia de riesgo cardiovascular en niños y adolescentes con sobrepeso y obesidad, por género y grupos de edad. Diseño: Estudio descriptivo, transversal. Participantes: Niños y adolescentes, de ambos géneros, comprendidos entre los 5 a 18 años. Intervención: El diagnóstico de sobrepeso y obesidad se hizo de acuerdo a la clasificación de Must y col. con los siguientes criterios: de 85 a 95 para el sobrepeso y más de 95 percentil obesidad. En sangre se determinó el perfil lipídico, glucosa e insulina; con estas dos últimas se determinó el índice HOMA, para identificar resistencia a la insulina (RI), mediante la ecuación de Matthew, el sindrome metabólico (SM) con la referencia de Cook y las dislipidemias (D) con las sugerencias de Friedman y de Daniels. Principales medidas de resultados: Riesgo cardiovascular. Resultados: Los obesos presentan mayores riesgos cardiovasculares que los que tienen sobrepeso: RI 77,8\%, SM 22,2\%; D: colesterol total (CT) 64,4\%, C-HDL 33,3\%, C-LDL 19,5\% y triglicéridos $(\mathrm{Tg}) 40 \%$. El género masculino, independientemente de su estado nutricional, tuvo mayor riesgo que el femenino: RI 74,1\%, SM 22,2\%, D: CT 63\%, C-HDL 37\%, C-LDL 36\% y Tg 40,7\%. En cuanto a la edad, en los mayores de 10 años se ha encontrado $\mathrm{Rl}$ en $78 \%$ y C-HDL 31,7\%; los demás factores de riesgo fueron mayores en los menores de 10 años. Conclusiones: Los riesgos afectaron más a los obesos que a los que tenían sobrepeso, más al género masculino y, en cuanto a la edad, los mayores de 10 años presentaron mayor prevalencia de RI y de C-HDL bajo. A mayor IMC más riesgo cardiovascular, lo que compromete muy seriamente la salud y que se hace más preocupante porque su aparición es a temprana edad.
\end{abstract}

Palabras clave: Niños y adolescentes; sobrepeso y obesidad; riesgo cardiovascular.

Abstract

Introduction: Overweight and obesity lead the occurrence of comorbidities such as type 2 diabetes mellitus, hypertension, and dyslipidemias. Objectives: To determine the presence of cardiovascular risk in children and adolescents overweight and obese, by gender and age groups. Design: Cross sectional study. Participants: Children and adolescents of both genders, 5-18 year-old. Interventions: Diagnosis of overweight and obesity was done according to Must et al. classification with the following criteria: 85 to 95 for overweight and more than 95 percentile for obesity. Serum lipid profile, glucose and insulin were analyzed, and with the latter two HOMA index was determined to identify insulin resistance (IR), using Matthew's equation, the metabolic syndrome (MS) with reference to Cook and dyslipidemias (D) with Friedman and Daniels' suggestions. Main outcome measures: Cardiovascular risk. Results: Obese children had higher cardiovascular risks than overweight: IR $77.8 \%$, SM 22.2\%, D: total cholesterol (TC) $64.4 \%$, HDL-C 33.3\%, LDL-C $19.5 \%$ and triglycerides $(\mathrm{Tg})$ 40\%. Males had higher risk than females regardless of nutritional status: IR $74.1 \%$, SM $22.2 \%$, D: TC $63 \%$, HDL-C 37\%, LDL-C 36\%, Tg 40.7\%. Children over 10 had IR 78\% and HDL-C 31.7\%; other risk factors were higher in those under 10 years. Conclusions: Risks affected obese more than overweight children, more to males, and those over 10 years had higher prevalence of IR and low HDL-C. With higher BMI more cardiovascular risk and serious health compromise, worrisome because of early age onset.

Keywords: Children and adolescents, overweight and obesity, cardiovascular risk.

An Fac med. 2013;74(3):181-6

\section{INTRODUCCIÓN}

En 1980, Khuory ${ }^{(1)}$, en el Cincinnati Lipid Research Clinics Princenton Study, fue quizás el primero en llamar la atención de la presencia de factores de riesgo cardiovascular en niños de 6 a 19 años. Posteriormente. Smoak (2), en 1987, en el Bogalusa Heart Study, comunicó lo mismo, pero en niños obesos.
En el Perú, en adolescentes con sobrepeso y obesidad, se ha publicado estudios que muestran la presencia de factores de riesgo cardiovascular, como el síndrome metabólico (SM), en 3 y $22,9 \%{ }^{(3)}$, resistencia a la insulina (RI) 12,3 y $16,2 \%{ }^{(4)}$, y posteriormente 20,3 y $27,4 \%{ }^{(5)}$. Otro artículo indica que la RI es más prevalente en los obesos ${ }^{(6)}$.
En cuanto a las dislipidemias, la hipercolesterolemia, 11,7 y $21,9 \%$, y la hipertrigliceridemia, 18,2 y $18,8 \%$, aparecen como las más prevalentes ${ }^{(7)}$. Todos estos estudios fueron realizados en centros educativos estatales.

Muchas de las complicaciones metabólicas y cardiovasculares de la obesidad ya están presentes durante la 
infancia y se encuentran fuertemente relacionadas a la RI, que es una de las anormalidades más comunes de la obesidad ${ }^{(8)}$. Existen muchas evidencias de que la obesidad en los niños crea una especie de plataforma metabólica para las enfermedades cardiovasculares en la adultez ${ }^{(9)}$.

De todas maneras, hay un impacto del incremento del índice de masa corporal (IMC) sobre ciertos parámetros cardiometabólicos; estos cambios no han sido muy grandes en lo que se refiere a la glicemia, pero sí bastante importantes en la prueba de tolerancia a la glucosa, la insulina, los triglicéridos y la presión arterial sistólica ${ }^{(10)}$. Sin embargo, se ha observado que los obesos con una prueba de tolerancia a la glucosa alterada son significativamente más insulino-resistentes que aquellos con una prueba normal, a pesar de tener igual grado de adiposidad. Estas diferencias en la sensibilidad a la insulina fueron atribuidas a la capacidad lipolítica de las grasas; aquellos que tienen marcada resistencia a la insulina se caracterizan por un depósito incrementado tanto en el compartimiento visceral como en el intramiocelular ${ }^{(11)}$.

La disfunción endotelial, los estados pro-inflamatorios y el estrés oxidativo son los elementos responsables, en el obeso, para incremento en su riesgo cardiovascular. Muchos de los trastornos proinflamatorios y proaterogénicos asociados con enfermedades cardiovasculares han también sido demostrados en niños obesos ${ }^{(12)}$.

El objetivo del presente estudio fue determinar la presencia de riesgo cardiovascular en niños y adolescentes con sobrepeso y obesidad, por género y grupos de edad.

\section{MÉTODOS}

Se estudió 58 niños y adolescentes, en un rango de edad de 5 a 18 años, 22,4\% con sobrepeso $(n=13)$ y $77,6 \%$ obesos $(n=45)$. El 46,6\% $(n=27)$ fue del género masculino y $53,4 \%(n=31)$ del femenino, $29,3 \%(n=17)$ menor de 10 años y $70,7 \%(n=41)$ de 10 años y más. Fueron atendidos en el Servicio de Endocrinología del Hospital Nacional Dos de Mayo, en el período comprendido entre mayo de 2010 a mayo de 2011. El criterio de inclusión fue que solo consultasen por su problema de peso, y como criterio de exclusión que este incremento de peso respondiese a problemas endócrinos o que presentasen una comorbilidad, como diabetes mellitus tipo 2, dislipidemias y/o hipertensión arterial.

Se les tomó el peso y la talla para determinar el IMC $(\mathrm{kg} / \mathrm{m} 2)$, ambas medidas siguiendo las recomendaciones internacionalmente aceptadas ${ }^{(13)}$. Además, se midió la circunferencia de la cintura (CC) en el punto medio de la línea axilar, que va desde la última costilla hasta la espina ilíaca ${ }^{(14)}$.

En sangre de venopuntura se determinó insulina basal, glucosa basal y perfil lipídico. Con las dos primeras se determinó el HOMA-I, de acuerdo a la ecuación de Matthew (insulina uUI/mL $\mathrm{x}$ glucosa mmol $/ 22,5)^{(15)}$. En el caso que el paciente respondiese afirmativamente si tenía familiares diabéticos, se le indicaba una prueba de tolerancia a la glucosa.

El diagnóstico nutricional se hizo con la población referencial de Must y col. ${ }^{(16)}$, con valores de IMC entre el 85 y 95 p para sobrepeso y los mayores de 95p obesidad. La identificación de las dislipidemias se hizo con CT $>=170$ $\mathrm{mg} / \mathrm{dL}, \mathrm{Tg}>=150 \mathrm{mg} / \mathrm{dL}, \mathrm{C}-\mathrm{HDL}$ $<=35 \mathrm{mg} / \mathrm{dL}$ y $\mathrm{C}-\mathrm{LDL}>=130 \mathrm{mg} /$ $\mathrm{dL}^{(17,18)}$. En el caso del SM, se utilizó los criterios recomendados por Cook ${ }^{(19)}$ : $\mathrm{Tg}>=110 \mathrm{mg} / \mathrm{dL}, \mathrm{C}-\mathrm{HDL}<=40$ $\mathrm{mg} / \mathrm{dL}$, glicemia $>=110 \mathrm{mg} / \mathrm{dL}$ y CC $>=90 \mathrm{p}$. Para identificar el SM fue necesario que la misma persona presentase tres o más de estos criterios; no se midió la presión arterial. El criterio diagnóstico para definir RI fue tener un HOMA-I $>=3,1^{(20)}$.

Las variables continuas fueron presentadas como medidas de tendencia central (media), de dispersión (desviación estándar) y con un intervalo de confianza (IC) de 95\%. Con la finalidad de comparar las medias, se utilizó la prueba $t$ de student en variables con distribución normal, y la prueba U de Mann Whitney en variables sin distribución normal. Se trabajó con un nivel de significancia de 0,05. Para relacionar el HOMA-I con las variables antropométricas y bioquímicas se usó la correlación de Spearman. En el caso de las tablas de contingencia, se utilizó el chi cuadrado. Se usó el paquete estadístico del SPSS 17.0.

Tabla 1. Medidas de tendencia central, dispersión y significancia estadística de las diferentes variables estudiadas según estado nutricional.

\begin{tabular}{|c|c|c|c|c|c|c|c|}
\hline \multirow{2}{*}{ Variables } & \multicolumn{3}{|c|}{ Sobrepeso $(n=13)$} & \multicolumn{3}{|c|}{ Obesidad $(n=45)$} & \multirow{2}{*}{$p$} \\
\hline & Media & D.E. & IC 95\% & Media & D.E. & IC $95 \%$ & \\
\hline Edad (años) & 14,2 & 3,4 & $12,2-16,3$ & 12,1 & 3,9 & $10,9-13,2$ & 0,074 \\
\hline Peso (kg) & 55,3 & 11,5 & $48,4-62,3$ & 72,1 & 25,5 & $64,4-79,7$ & 0,026 \\
\hline Talla (cm) & 145,2 & 10,7 & $138,7-151,7$ & 147,5 & 14,8 & $143,1-151,9$ & 0,612 \\
\hline IMC $\left(\mathrm{kg} / \mathrm{m}^{2}\right)$ & 25,9 & 2,2 & $24,6-27,2$ & 32 & 6,6 & $30-34$ & $<0,001$ \\
\hline $\mathrm{CC}(\mathrm{cm})$ & 86,1 & 6,8 & $82-90,2$ & 98,5 & 15,2 & $93,9-103,1$ & $<0,001$ \\
\hline Glucosa (mg/dL) & 86,8 & 7,4 & $82,3-91,3$ & 87,8 & 8,8 & $85-90,3$ & 0,745 \\
\hline Insulina (uUl/mL) & 13,9 & 5,6 & $10,5-17,3$ & 24,4 & 13,3 & $20,4-28,4$ & 0,008 \\
\hline HOMA-I & 3,0 & 1,2 & $2,3-3,7$ & 5,3 & 3,0 & $4,4-6,2$ & 0,009 \\
\hline CT (mg/dL) & 169,7 & 31,5 & $146,5-190$ & 177,3 & 27,5 & $169-187,4$ & 0,399 \\
\hline C-HDL (mg/dL) & 48,6 & 17,3 & $40,7-63,1$ & 40,7 & 10,0 & $38,5-44,7$ & 0,039 \\
\hline C-LDL (mg/dL) & 89,3 & 37,5 & $92,1-146,3$ & 94,9 & 37,5 & $83,6-106,2$ & 0,663 \\
\hline $\operatorname{Tg}(\mathrm{mg} / \mathrm{dL})$ & 119,2 & 44,8 & $83,7-139,4$ & 165,4 & 103,3 & $127-193,4$ & 0,222 \\
\hline
\end{tabular}




\section{RESULTADOS}

El grupo de los obesos presentó mayores promedios de peso $(p=0,02)$, de IMC $(p<0,001)$ y de la CC $(p=<0,001)$. El promedio de edad fue mayor en aquellos con sobrepeso mientras que la talla fue ligeramente mayor en los obesos, pero estas diferencias no fueron estadísticamente significativas (tabla 1).

Dentro de las variables bioquímicas, los obesos presentaron mayores promedios de insulina $(\mathrm{p}<0,001)$, HOMA-I $(\mathrm{p}<0,001)$ y glucosa, pero en esta última no hubo diferencia significativa $(p=0,745)$. En lo que respecta a los lípidos, el CT, C-LDL y Tg fueron más altos en los obesos, aunque no alcanzó significancia estadística. Por otro lado, el grupo con obesidad tuvo menores niveles de C-HDL, siendo esta última variable la única con significancia estadística $(\mathrm{p}=0,039)$.

En la tabla 2 se puede observar que la mayor prevalencia estuvo dada por la RI $(70,7 \%)$, siendo el grupo obeso el más afectado $(77,8 \%)$ en relación a los con sobrepeso $(46,2 \%)$. En cuanto al $\mathrm{SM}$, el problema fue menor $(17,2 \%)$ y solo se presentó en los obesos $(22,2 \%)$. Las dislipidemias más prevalentes fueron la hipercolesterolemia $(62,1 \%)$ y la hipertrigliceridemia $(36,2 \%) \mathrm{y}$, a excepción del C-LDL, las otras dislipidemias se las encontró más en los obesos. En lo que concierne al género, el masculino tuvo las mayores prevalencias en todos los factores de riesgo, respecto al femenino. En cuanto a la edad, los mayores estuvieron más afectados con la RI (78\%) y el C-HDL $(31,7 \%)$, mientras que los menores mostraron hipercolesterolemia $(76,5 \%)$ y la hipertrigliceridemia $(47,1 \%)$.

En la tabla 3 se puede observar los niveles de correlación del HOMA-I con las variables antropométricas, bioquímicas y con la edad. Como se puede observar, el HOMA-I presentó una correlación positiva con el peso, la talla, el IMC y la CC, siendo significativa estadísticamente $(\mathrm{p}<0,001)$ para cada una de ellas. En lo que se refiere a las variables bioquímicas, la más alta
Tabla 2. Prevalencias de factores de riesgo de acuerdo a estado nutricional, género y edad.

\begin{tabular}{|c|c|c|c|c|c|c|c|}
\hline Variables & $\mathrm{n}$ & RI & SM & CT & C-HDL & C-LDL & TG \\
\hline \multicolumn{8}{|l|}{ Estado nutricional } \\
\hline Sobrepeso & 13 & 46,2 & - & 53,8 & 23,1 & 27,3 & 23,1 \\
\hline Obesidad & 45 & 77,8 & 22,2 & 64,4 & 33,3 & 19,5 & 40 \\
\hline \multicolumn{8}{|l|}{ Género } \\
\hline Masculino & 27 & 74,1 & 22,2 & 63 & 37 & 36 & 40,7 \\
\hline Femenino & 31 & 67,7 & 12,9 & 61,3 & 25,8 & 7,4 & 32,3 \\
\hline \multicolumn{8}{|l|}{ Grupo etáreo } \\
\hline Edad $<10$ años & 17 & 52,9 & 23,5 & 76,5 & 29,4 & 28,6 & 47,1 \\
\hline Edad $>=10$ años & 41 & 78 & 14,6 & 56,1 & 31,7 & 18,4 & 31,7 \\
\hline Totales & 58 & 70,7 & 17,2 & 62,1 & 31 & 21,2 & 36,2 \\
\hline
\end{tabular}

correlación estuvo dada con la insulina $(p<0,001)$. Las otras no presentaron significación estadística.

El cruce de variables como el SM y la RI muestran que $27,6 \%$ de la población estudiada se encontró exento de estos factores, mientras que $15,5 \%$ presentó ambos factores de riesgo. Tanto la prueba de chi cuadrado como la estimación de riesgo no mostraron significación estadística (tabla 4).

En la tabla 5 se muestra la razón de prevalencia entre la RI y el estado nutricional, género y grupo etario, observándose que el mayor riesgo de presentar RI fue en los obesos, de género masculino y que tenían mayor edad; sin embargo, estas diferencias no presentaron significación estadística.

\section{DISCUSIÓN}

Tradicionalmente, el Hospital Dos de Mayo ha atendido a pacientes adultos; esto explicaría que el número de población estudiada sea tan pequeño.

Tabla 3. Niveles de correlación del HOMA-I con las variables antropométricas y bioquímicas.

\begin{tabular}{ccc} 
Variables & $\begin{array}{c}\text { Nivel de } \\
\text { correlación }\end{array}$ & $p$ \\
Edad & 0,239 & 0,06 \\
Peso & 0,564 & $<0,001$ \\
Talla & 0,407 & 0,002 \\
IMC & 0,65 & $<0,001$ \\
CC & 0,53 & $<0,001$ \\
Glucosa & 0,056 & 0,677 \\
Insulina & 0,983 & $<0,001$ \\
CT & 0,034 & 0,8 \\
C-HDL & $-0,199$ & 0,133 \\
C-LDL & 0,054 & 0,703 \\
Tg & 0,338 & 0,01 \\
\hline
\end{tabular}

La RI está considerada como uno de los factores de riesgo más comunes en la obesidad ${ }^{(8)}$. Un estudio realizado en un colegio, con un grupo de adolescentes, encontró una prevalencia de RI de $20,3 \%$ en los con sobrepeso, mientras que alcanzaba $27,4 \%$ en los obesos ${ }^{(5)}$. En el presente estudio, las prevalencias fueron mucho mayores (sobrepeso

Tabla 4. Prevalencia de niños y adolescentes, con sobrepeso y obesidad, con la presencia o no de RI y SM.

\begin{tabular}{ccccc} 
& & \multicolumn{2}{c}{ Resistencia a la insulina } & \multirow{2}{*}{ Total } \\
\cline { 2 - 4 } & & Sí & No & \\
Síndrome & Sí & 15,5 & 1,7 & 17,2 \\
\cline { 2 - 4 } metabólico & No & 55,2 & 27,6 & 82,8 \\
\hline Total & & 70,7 & 29,3 & 100 \\
\hline
\end{tabular}


Tabla 5. Razón de prevalencia de resistencia a la insulina según estado nutricional, género y grupo etario.

\begin{tabular}{ccccc} 
Variables & \multicolumn{5}{c}{ Resistencia a la insulina } \\
\cline { 2 - 5 } & $\mathrm{n}$ & $\mathrm{RP}$ & $\mathrm{IC} 95 \%$ & $\mathrm{p}$ \\
\hline Estado nutricional & & & & \\
\hline $\begin{array}{c}\text { Sobrepeso } \\
\text { Obesidad }\end{array}$ & 13 & 1 & Ref. \\
Género & 45 & 1,68 & $0,93-3,09$ & 0,092 \\
\hline Femenino & 31 & 1 & Ref. & \\
Masculino & 27 & 1,09 & $0,79-1,52$ & 0,595 \\
\hline Grupo etáreo & & & & \\
Edad $<10$ años & 17 & 1 & Ref. & \\
Edad $>=10$ años & 41 & 1,47 & $0,92-2,38$ & 0,11 \\
\hline
\end{tabular}

RP: Razón de prevalencia.

$46,2 \%$ y obesidad $77,8 \%)$. Al comparar estos datos daría la impresión que las prevalencias se han incrementado; pero, en realidad lo que sucede es que los que acuden al hospital serían como una población que ya presenta alguna complicación de tipo mecánica, lo que obligaría a que sus padres los lleven a la consulta, lo que no debe suceder con el otro grupo. Independientemente de las prevalencias, lo que sí se aprecia es que conforme se incrementa el IMC aumenta la presencia de RI.

Un estudio llevado a cabo en Turquía, con niños de 7 a 18 años obesos (IMC > 95p) que asistían al Departamento de Endocrinología Pediátrica, encontró una prevalencia de 40,2\% de niños con RI (HOMA-I $>=3,16$ ), más en los pospúberes $(56,5 \%)$ que en los púberes (29\%) ${ }^{(21)}$. Cifras más o menos similares fueron encontradas en España, con $35,8 \%{ }^{(22)}$. La mayor presencia de RI en el grupo pospúber también se observa en nuestro estudio (78 versus $52,9 \%)$.

En un Centro Pediátrico de Cochabamba-Bolivia, en niños obesos, un $39,4 \%$ tuvo RI (HOMA-I > 3,5), siendo los de género masculino los más afectados $(50 \%)$ en relación al femenino $(29 \%)^{(23)}$. Esta diferencia a favor del género masculino también se ha observado en el presente estudio (74,1 versus $67,1 \%)$. En los EE UU mostraron la relación que existe entre el estado nutricional y la RI, normal 0,3\%, sobrepeso $5,9 \%$ y obesidad $35,1 \%$, con un HOMA-I > 3,99 ${ }^{(24)}$. De la misma manera, Lee y col. encontraron que 9, 20 y $60 \%$ de niños normales, con sobrepeso y obesidad, presentaron RI (HOMA-I $>3,99)^{(25)}$. En niños mexicanos se ha hallado 90\% de IR en obesos (HOMA-I $>3,1)^{(26)}$. La relación estrecha entre la obesidad y la RI también ha sido demostrada utilizando como criterio diagnóstico valores que se encuentran por encima del tercer tertil de la misma población estudiada ${ }^{(27)}$.

Todos los estudios revisados han utilizado el HOMA-I y con un criterio diagnóstico más o menos semejante para identificar la RI. Los estudios transversales tienen como limitación que no proporcionan información del tiempo en que se tiene el sobrepeso o la obesidad y menos desde cuándo se tiene la RI.

La RI está considerada como una prediabetes. El 31\% (18/58) de la población estudiada refirió tener algún familiar o familiares con diabetes mellitus. En ellos se indicó una prueba de tolerancia oral a la glucosa, detectándose a dos niños con esta patología, ambos de 15 años y con diagnóstico de obesidad. Lo curioso es que un adolescente tenía un IMC de 30,8 kg/m2, insulina de 9,57 $\mathrm{uUI} / \mathrm{mL}$ y HOMA-I de 2,15, mientras que en el otro los valores eran $52 \mathrm{~kg} /$ m2, 12 de insulina y 2,7 de HOMA-I, o sea que no presentaban ni hiperinsulinemia ni RI y por ende se podría decir que las causales de su diabetes mellitus tipo 2 fueron la obesidad y sus antecedentes familiares y no la RI.

Sin embargo, el tener sobrepeso y obesidad presentó una asociación con la RI (chi cuadrado 4,8 p < 0,05 IC95\% 1,11 a 1,45). Para las otras relaciones, como son el SM y los diferentes lípidos, no existió significación estadística. De alguna manera, esto podría explicar las correlaciones estadísticamente significativas que tiene el HOMA-I con los indicadores antropométricos (peso, talla, IMC y CC), más no con los lípidos. Es de destacar que, en los que presentan RI, sus niveles de glucosa permanecen normales y esto podría deberse a una hiperinsulinemia compensatoria y una adecuada reserva pancreática de células beta ${ }^{(28)}$.

El ser obeso, pertenecer al género masculino y ser mayor de 10 años conlleva mayor riesgo de RI que el tener sobrepeso, ser del género femenino y menor de 10 años; pero esta diferencia no es significativa y eso quizás se deba a que el $n$ es reducido y que no se ha trabajado ajustando las variables.

Otra situación que se presenta es el SM, que es una entidad bastante controversial. Dentro de los problemas que se debe enfrentar es la existencia de muchas poblaciones de referencia para la $\mathrm{CC}$, donde en algunas es el criterio básico, mientras que para otras es uno más. Pero, lo que realmente tiene mayor repercusión son los valores diferentes para el 90 percentil que es el criterio diagnóstico de riesgo. Es por esta razón que se debe tener cautela cuando uno compara prevalencias y lo recomendable es hacerlo utilizando las mismas poblaciones de referencia.

En China, con los criterios de la International Diabetes Federation (IDF), encontraron $0,8 \%$ de niños mayores de 10 años con SM, mientras que con la de Cook fue $2,5 \%{ }^{(29)}$. Independientemente de la referencia que se use, la tendencia 
es que las mayores prevalencias las presentan los obesos $(6,6 \%)$, que los con sobrepeso $(0,9)$ y los normales $(0 \%)$. En el presente estudio se tuvo $22 \%$ de SM en obesos y se afirma que las diferentes prevalencias encontradas pudieran ser debidas a las diferentes prevalencias de obesidad. En el estudio chino, la obesidad alcanzó 10,3\%. La mayor presencia de SM en la obesidad en relación a los que tienen sobrepeso ya fue señalado anteriormente ${ }^{(3)}$.

Morrison (30) encontró que de los niños con SM el 68,5\% puede tener el SM en la adultez y que $15,6 \%$ podría desarrollar diabetes mellitus tipo 2, 25 a 30 años después, mientras que las proporciones de los que no tienen SM serían 24 y $5 \%$, respectivamente.

Existen opiniones diversas en cuanto al diagnóstico y a la utilidad del SM. El estudio de Van Guilder, realizado en personas normales (IMC), obesos sin SM y obesos con SM, muestra una mayor presencia de marcadores biológicos de estrés oxidativo e inflamación (LDL oxidada, factor de necrosis tumoral, interleuquina 6 y 18 y proteína $\mathrm{C}$ reactiva) a favor de estos últimos, siendo esta diferencia estadísticamente significativa ${ }^{(31)}$.

Si bien el sobrepeso y la obesidad traen aparejados una serie de riesgos, esto no es concluyente ni universal. En ese sentido existe un $27,6 \%$ de pacientes que no presenta RI ni SM y que son los llamados obesos metabólicamente normales, de acuerdo a lo propuesto por Meiggs ${ }^{(32)}$. Esto quizás podría responder a factores genéticos; lamentablemente, los estudios de genética son muy escasos y por esa razón no se puede asegurar nada; mientras tanto hay que seguir luchando en el campo de la prevención del sobrepeso y la obesidad.

En lo que se refiere a las dislipidemias, existen realidades diferentes; así se tiene que en áreas rurales de Túnez las prevalencias son bajas (CT 2,9, C-HDL 0,8, C-LDL 0,6 y Tg 1\%, respectivamente), siendo los criterios diagnósticos usados similares a los del presente trabajo, a excepción de los $\mathrm{Tg}$
(>170 mg/dL) ${ }^{(33)}$. Prevalencias mucho mayores han sido observadas en niños mexicanos, concluyendo que la obesidad es un riesgo de dislipidemias (34). Así mismo, en la India, los obesos presentan mayores promedios de los lípidos que los de su grupo control (35). Las características de las dislipidemias encontradas en nuestro estudio es que las mayores prevalencias estuvieron dadas por la hipercolesterolemia $(62,1 \%)$, seguido por la hipertrigliceridemia $(36,2 \%)$. Mayores prevalencias se presentaron en el género masculino, en el grupo de menores de 10 años y en los obesos. En un estudio anterior, realizado en adolescentes del género femenino, se encontró la misma tendencia ${ }^{(7)}$. Estas anormalidades en los lípidos tienen importante rol en el desarrollo de enfermedades coronarias en la adultez; estas enfermedades son la mayor causa de morbilidad y mortalidad tanto en los países desarrollados como en los no desarrollados ${ }^{(36)}$.

Estudios de autopsias, tales como el Phatobiological Determinants of Atherosclerosis in Youth (37) y el Bogalusa Heart Study han demostrado que los procesos ateroscleróticos empiezan a temprana edad ${ }^{(38)}$. El Cardiovascular Risk in Young Finns Study mostró una relación entre factores de riesgo en adolescentes y aterosclerosis en los adultos ${ }^{(39)}$.

La razón de la presencia de todos estos problemas se debe a los fenómenos de transición que se vienen dando en el mundo. El proceso de cambio de los estilos de vida está generando que el sobrepeso y la obesidad se incrementen y por consecuencia sus comorbilidades. Es por eso que se debe tomar medidas de tipo preventivo, como promover la actividad física y una alimentación saludable. Esta medidas tienen efectos independientes sobre la función endotelial, pero que se potencializan cuando se las trabaja en forma coordinada ${ }^{(40)}$.

En conclusión, se puede observar que los riesgos afectan más a los obesos que a los que tienen sobrepeso, más al género masculino; y en cuanto a la edad, los mayores de 10 años presen- tan más prevalencia de RI y de C-HDL bajo. A mayor IMC más riesgo cardiovascular, lo que compromete muy seriamente la salud, y que se hace más preocupante porque su aparición es a temprana edad.

\section{REFERENCIAS BIBLIOGRÁFICAS}

1. Khuory P, Morrison A, Kelly K, Horvitz R, Glueck C. Clustering and interrelationships of coronary heart disease risk factors in schoolchildren ages 6-9. Am J Epidemiol. 1980;112:524-38.

2. Smoak C, Burke G, Webber L, Harsha D, Srinivasan $\mathrm{S}$, Berenson $\mathrm{G}$. Relation of obesity to clustering of cardiovascular disease risk factor in children and young adults. Am J Epidemiol. 1987;125:364-72.

3. Pajuelo J, Bernui I, Peña A, Zevillano L. Prevalencia de sindrome metabólico en adolescentes con sobrepeso y obesidad. An Fac med. 2007;68(2):143-7.

4. Pajuelo J, Pando R, Leyva M, Hernández K, Infantes R. Resistencia a la Insulina en adolescentes con sobrepeso y obesidad. An Fac med. 2006;67(1):23-9.

5. Pajuelo J, Bernui I, Rocca J, Torres L, Soto I. Marcadores bioquímicos de riesgo cardiovascular en una población adolescente femenina con sobrepeso y obesidad. An Fac med. 2009;70;1:7-10.

6. Rojas MI, Nuñez O, Del Aguila C, Briceño M, Valenzuela N. Resistencia a insulina en adolescentes obesos. An Fac med. 2010;71(1):13-7.

7. Pajuelo J. Los lípidos en adolescentes con sobrepeso y obesidad. X Congreso Nacional de Alimentación y Nutrición. Sociedad Peruana de Nutrición. Lima 2011

8. Lee J, Okumura M, Davis M, Herman W, Gurney J. Prevalence and determinants of insulin resistance among US adolescents. A population-based study. Diabetes Care. 2006;29:2427-32.

9. Grundy S. Obesity, metabolic syndrome, and coronary aterosclerosis. Circulation. 2002;105:2696-8.

10. Cali A, Caprio S. Obesity and children and adolescents. J Clin Endocrinol Metab. 2008;93(11):S31S36.

11. Wiess R, Dufour S, Taksali S, Tamborlane W, Petersen K. et al. Prediabetes in obese youth a syndrome of impaired glucose tolerance, severe insulin resistance, and altered myocelullar and abdominal fat partitioning. Lancet. 2003;362:951-7.

12. Montero D, Walther G, Perez-Martin A, Roche E, Vinet A. Endothelial dysfunction, inflammation, and oxidative stress in obese children and adolescents: markers and effect of lifestyle intervention. Obesity Rev. 2012;13:441-55.

13. Lohman T, Roche A, Martorell R. Anthropometric Standardization Reference Manual. Human Kinetics Books. 1988.

14. Wang J, Thorton J, Bari S, Williamson B, Gallagher D, Heymsfield S, et al. Comparisons of waist circumference measured at 4 sites. Am J Clin Nutr. 2003;77:379-84.

15. Mattews D, Hosker J, Rudenski A, Naylor B, Teacher D, Turner R. Homeostasis model assessment insulin resistance and cell function from fasting plasma glucose and insulin concentration. Diabetologia. 1985;28:412-9. 
16. Must A, Dallal G, Dietz W. Reference data for obesity: 85th and 95th percentiles of body mass index (wt/ht2)-a correction. Am J Clin Nutr. 1991;54:773.

17. Daniels S, Greer F; Committee on Nutrition. Lipid screening and cardiovascular health in childhood. Pediatrics. 2008;122:198-208.

18. Friedman L, Morrison A, Daniels S, McCarthy W, Sprecher D. Sensitivity and specificity of pediatric lipid determinations for adult lipid status: findings from the Princeton Lipid Research Clinics Prevalence Program Follow-up Study. Pediatrics. 2006;118(1):165-72.

19. Cook S, Weitzman M, Auinger P, Nguyen M, Dietz W. Prevalence of a metabolic syndrome phenotype in adolescents: findings from the third National Health and Nutrition Examination Survey, 1988-1994. Arch Pediatr Adolesc Med. 2003;157:821-7.

20. Keskin M, Kurtoglu S, Kendirci M, Atabek ME, Yazici C. Homeostasis model assessment is more reliable than the fasting/glucose ratio and quantitative insulin sensitivity check index for assessing insulin resistance among obese children and adolescents. Pediatrics. 2005;115:500-3.

21. Atabek M, Pirgon O, Kurtoglu S. Prevalence of metabolic syndrome in obese Turkish children and adolescents. Diabetes Res Clin Pract. 2006;72:315-21.

22. Tresaco B, Bueno G, Moreno L, Garagorria M, Bueno $M$. Insulin resistance and impaired glucose tolerance in obese children and adolescents. J Physiol Biochem. 2003;59:217-23.

23. Caceres M, Teran C, Rodriguez S, Medina M. Prevalence of insulin resistance and its association with metabolic syndrome criteria among Bolivian children and adolescents with obesity. BMC Pediatrics. 2008;8;31-6.

24. Turchiano M, Sweat V, Fierman A, Convit A. Obesity, metabolic syndrome and insulin resistance in urban high school students of minority race/ethnicity. Arch Pediatr Adolesc Med. 2012;166(1):1030-6.
25. Lee J, Bacha F, Gungor N, ArslanianS. Comparison of different definitions of pediatric metabolic syndrome: relation to abdominal adiposity, insulin resistance, adiponectin, and inflammatory biomarkers. J Pediatr. 2008;152(2):177-84

26. Perichart-Perera O, Balas-Nakash M, SchiffmanSlechnick E, Barbato-Dosal A, Vadillo-Ortega F. Obesity increases metabolic syndrome risk factor in school-aged children from an urban school in Mexico City. J Am Diet Assoc. 2007;107:81-91.

27. Dhuper S, Cohen H, Daniel J, Gumidyala P, Agarwalla V, StVictor R, Dhuper S. Utility of the modified ATP III defined metabolic syndrome and severe obesity as predictors of insulin resistance in overweight children and adolescents: a crosssectional study. Cardiovascular Diabetology. 2007;6:14-9.

28. Nelson R, Bremer A. Insulin resistance and metabolic syndrome in the pediatric population. Metab Syndr Relat Disord. 2010;8(1):1-14.

29. Xu H, Li Y, Liu A, Zhang Q, Hu X, Fang $H$, et al. Prevalence of the metabolic syndrome among children from six cities of China. BMC Public Health. 2012;12:13.

30. Morrison J, Friedman L, Wang P, Glueck C. Metabolic syndrome in childhood predicts adult metabolic syndrome and type 2 diabetes mellitus 25-30 years later. J Pediatr. 2008:152(2):201-6.

31. Van Guilder G, Hoetzer G, Greiner J, Stanfer B, De Souza C. Influence of metabolic syndrome on biomarkers of oxidative stress and inflammation in obese adults. Obesity. 2006;14(12):2127-31.

32. Meigs J, Wilson P, Fox C, Vasan R, Nathan D, Sullivan $\mathrm{L}$, et al. Body mass index, metabolic sindrome and risk of type 2 diabetes or cardiovascular disease. J Clin Endocrinol Metab. 2006;91:2006-12.

33. Ghannem H, Harrabi I, Ben Abdelazari A, Gah R, Trabelsi R, Selmi H. Risque cardiovasculaire chez lenfant en milieu scolaire rural a Seusse, Tunisia. Arch Mal Coeur Vaiss. 2001;94(12):1981-5.

34. Romero E, Campollo O, Celis A y col. Factores de riesgo de dislipidemia en niños y adolescentes con obesidad. Salud Pública Mex. 2007;49(2):103-8.
35. Kaur S, Kapil U. Dyslipidemia amongst obese children in National Capital Territory (NCT) of Delhi. Indian J Pediatrica. 2011;78:55-75.

36. Lauer R, Lee J, Clarke W. Factors affecting the relationship between childhood and adult cholesterol levels: the Muscatines study. Pediatrics. 1988;82:309-18.

37. Berenson G, Srinivasas S, Bao W, Newman N, Tracy R, Wittignen W. Association between multiple cardiovascular risk factors and the early development of atherosclerosis. Bogalusa Heart Study. N Engl J Med. 1998;338(23:1650-56.

38. McGill H, McMahan C, Malcolm G, Oalmann M, Strong S. Effects of serum lipoproteins and smoking in atherosclerosis in young men and women. Pathobiological determinants of atherosclerosis in youth. Arterioscler Thromb Vasc Biol. 1998;17(1):95-102.

39. Raitakari O, Juonola M, Kahönen M, Taittonen $\mathrm{L}$, Laitinen $\mathrm{T}$, et al. Cardiovascular risk factors in childhood and carotid intima media thickness in adulthood: the Cardiovascular Risk in Young Finns Study. JAMA. 2003;290(17):2277-83

40. Woo KS, Chook P, Yu CW, Sug RY, Qiao, et al. Effects of diet and exercise on obesity-related vascular dysfunction in children. Circulation. 2004;109:1981-6.

Artículo recibido el 24 de febrero de 2013 y aceptado para publicación el 6 de mayo de 2013.

Los autores declaran que no existen conflictos de intereses.

Correspondencia:

Dr. Jaime Pajuelo Ramirez

Correo electrónico: japara18@yahoo.com 